\title{
Urinary soluble CD90 predicts renal prognosis in patients with diabetic kidney disease
}

\author{
Liang Wu $u^{1,2,3,4,5}$, Dong-Yuan Chang ${ }^{1,2,3,4,5}$, Lu-Xia Zhang ${ }^{1,2,3,4,5,6}$, Min Chen ${ }^{1,2,3,4,5}$, Ming-Hui Zhao ${ }^{1,2,3,4,5}$
}

${ }^{1}$ Renal Division, Department of Medicine, Peking University First Hospital, Beijing, China; ${ }^{2}$ Institute of Nephrology, Peking University, Beijing, China; ${ }^{3}$ Key Laboratory of Renal Disease, Ministry of Health of China, Beijing, China; ${ }^{4}$ Key Laboratory of Chronic Kidney Disease Prevention and Treatment (Peking University), Ministry of Education, Beijing, China; ${ }^{5}$ Research Units of Diagnosis and Treatment of Immune-mediated Kidney Diseases, Chinese Academy of Medical Sciences, Beijing, China; ${ }^{6}$ Center for Data Science in Health and Medicine, Peking University, Beijing, China Contributions: (I) Conception and design: LX Zhang, M Chen, MH Zhao; (II) Administrative support: M Chen; (III) Provision of study materials or patients: LX Zhang, M Chen, MH Zhao; (IV) Collection and assembly of data: L Wu, DY Chang; (V) Data analysis and interpretation: L Wu, DY Chang; (VI) Manuscript writing: All authors; (VII) Final approval of manuscript: All authors.

Correspondence to: Min Chen. Renal Division, Department of Medicine, Peking University First Hospital; Institute of Nephrology, Peking University; Key Laboratory of Renal Disease, Ministry of Health of China; Key Laboratory of Chronic Kidney Disease Prevention and Treatment (Peking University), Ministry of Education; Beijing 100034, China. Email: chenmin74@sina.com.

Background: Diabetic kidney disease (DKD), the major cause of chronic kidney disease, is associated with progressive renal fibrosis. The expression of CD90 correlated with fibrogenesis. However, the association between urinary soluble CD90 and renal disease severity, and whether it predicts outcomes in patients with DKD are still unclear.

Methods: Urinary sCD90 was measured in 285 patients with DKD in a longitudinal cohort. The composite endpoint was defined as end-stage renal disease (ESRD) or $40 \%$ reduction of estimated glomerular filtration rate (eGFR). The associations between urinary sCD90/Cr and clinical parameters, as well as renal outcomes were evaluated. Moreover, we detected the intrarenal CD90 expression, and demonstrated the correlation of intrarenal CD90 with clinico-pathological parameters.

Results: The urinary sCD90 level of DKD patients is significantly higher than diabetes patients without kidney injuries and healthy controls. We further showed urinary sCD90/Cr had significantly correlations with eGFR ( $\mathrm{r}=-0.373, \mathrm{P}<0.001)$, uACR $(\mathrm{r}=0.303, \mathrm{P}<0.001)$, serum creatinine $(\mathrm{r}=0.344, \mathrm{P}<0.001)$, and the eGFR slope $(\mathrm{r}=-0.27, \mathrm{P}<0.001)$. Elevated urinary sCD90/Cr was an independent risk factor for the composite endpoint, adjustment for potential confounders in DKD patients (HR 1.20, 95\% CI: 1.04-1.38, $\mathrm{P}=0.015$ ). However, the CD90 expression in the renal tubulointerstitial compartment in DKD patients was significantly lower than healthy controls, and showed significant negative correlations with the interstitial fibrosis and tubular atrophy score (IFTA) ( $\mathrm{r}=-0.3, \mathrm{P}=0.047)$, and urinary sCD90/Cr $(\mathrm{r}=-0.399, \mathrm{P}=0.029)$.

Conclusions: This study provided evidence that urinary sCD 90 could reflect the disease severity and serve as a valuable factor for renal outcome prediction in patients with DKD.

Keywords: CD90; diabetic kidney disease (DKD); estimated glomerular filtration rate slope (eGFR slope); endstage renal disease (ESRD)

Submitted Sep 22, 2020. Accepted for publication Nov 20, 2020.

doi: $10.21037 / \mathrm{atm}-20-6528$

View this article at: http://dx.doi.org/10.21037/atm-20-6528

\section{Introduction}

Diabetic kidney disease (DKD), the most common micro-vascular complication of diabetes mellitus (DM), is the leading cause of chronic kidney disease worldwide (1-3). It is also the major cause of end-stage renal disease (ESRD) (4). DKD originates from metabolic dysregulation. 
Inflammation, endothelial injuries, fibroblast abnormalities and macrophages infiltration further contribute to disease progression (5). Based on the key molecular mechanisms underlying DKD, discovering novel biomarkers for the accurate kidney disease progression prediction is of great relevance.

Cluster of differentiation 90 (CD90), also known as Thy-1, is a glycosylphosphatidyl-anchored protein of the immunoglobulin superfamily. CD90 is mainly expressed on the surface of cells, such as mesenchymal stem cells (6-10). It is also present as a soluble form (sCD90), which is an enzymatically cleaved ectodomain of CD90 (11). CD90 mediates different kinds of fundamental cellular processes, and is involved in the progression of several diseases (12). CD90 was reported to play the regulatory role in cell differentiation, especially in mesenchymal stromal cells (MSCs) and MSC-like cells. These regulatory roles have been established both in experimental models and various human diseases $(7,13,14)$. In particular, CD90 positive and CD90 negative mesenchymal fibroblasts showed different potential to differentiate into myofibroblasts and response to profibrotic cytokines. Dedifferentiation of tubular epithelial cells, perivascular MSC-like cells and pericytes contributes to further tubular injuries and renal fibrosis in DKD (15). Thus, whether sCD90 is associated with the disease severity and renal progression in DKD aroused our attention.

In this study, we evaluated the associations of sCD90 in urine and plasma with clinical parameters, and the prognostic value of sCD90 in predicting the progression of renal function in DKD. Moreover, the association between CD90 expression in kidneys and clinico-pathological parameters was assessed in patients with DKD. We present the following article in accordance with the STROBE reporting checklist (available at http://dx.doi.org/10.21037/ atm-20-6528).

\section{Methods}

\section{Patients recruitment}

DKD patients were recruited from the C-STRIDE cohort (16). Inclusion criteria have been described previously $(17,18)$. Complications with other known renal diseases were excluded. In total, we enrolled 285 DKD patients with regular follow-up to conduct the longitudinal study. We also collected urine and plasma samples from 29 DM patients without kidney injuries from the outpatient clinic of the Department of Endocrinology of our hospital. Patients with any other disease including connective tissue diseases, chronic kidney disease, hepatic dysfunction and malignancy, were excluded. In addition, patients who were on any concomitant medication other than antihyperglycemic drugs were excluded. Urine and plasma samples of 22 age- and sex-matched healthy donors were collected. These healthy controls did not have a history of hypertension, diabetes, cardiovascular diseases and renal diseases. None of them was under medical treatment. The blood and urine samples were treated as follows. The morning urine samples were pre-treated by centrifuge with $600 \mathrm{~g}$ and $5 \mathrm{~min}$. Then the samples were transferred to the $-80{ }^{\circ} \mathrm{C}$ refrigerator for further experimental tests. Blood samples in the ethylenediamine tetraacetic acid (EDTA) tubes were centrifuged at $3,000 \mathrm{~g}$ for $10 \mathrm{~min}$ at $4^{\circ} \mathrm{C}$. Then the blood samples were aliquoted and shifted into the $-80{ }^{\circ} \mathrm{C}$ refrigerator.

Kidney biopsy samples were obtained from 30 patients with biopsy-proven DKD in Peking University First Hospital during 2017. DKD was diagnosed by the characteristic of pathological features, as previously described (19). Patients accompanied by other co-existing renal diseases were excluded. During the renal biopsy, clinical and laboratory data acquisition were accomplished. The control kidney samples $(n=8)$ were from the healthy kidney poles of individuals, who received tumor nephrectomies without diabetes or other kidney diseases. All control kidney samples were confirmed by pathological examinations. The study was conducted in accordance with the Declaration of Helsinki (as revised in 2013). The study was approved by the Ethics Committee of Peking University First Hospital (Beijing, China) (No.: 2011[363]; 2017[1280]) and informed consent was taken from all the patients.

The recruitment details were shown in Figure 1.

\section{Renal histopathology}

The renal biopsy samples were detected and observed under light microscopy, fluorescent microscope and electron microscopy. Biopsy samples were evaluated by two pathology experts separately. These experts complied with the standard protocol for scoring renal biopsies of patients with DKD, and gave the final score within the blind method. The pathological findings of renal biopsies were classified as described previously (20). 
A

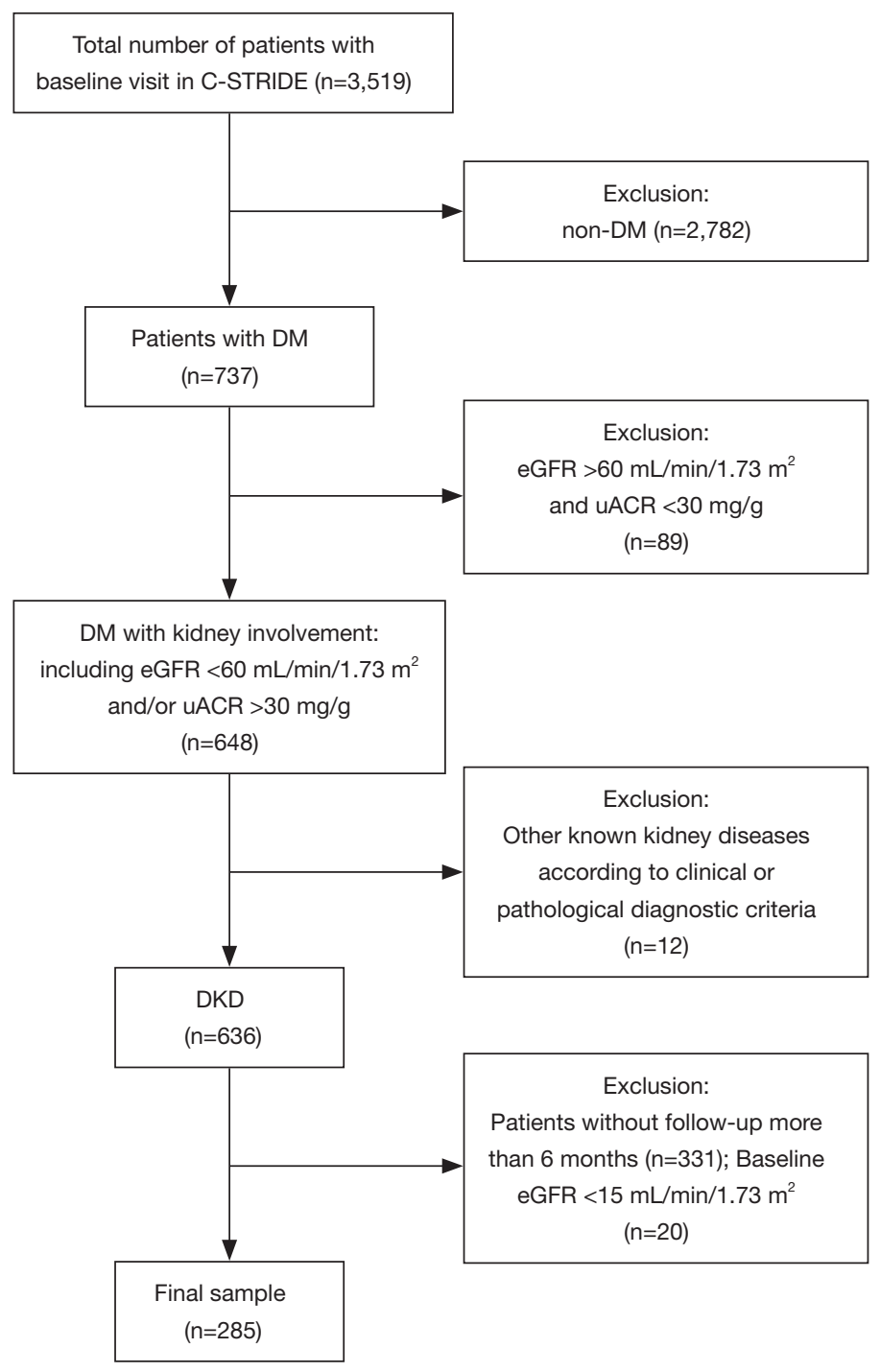

B

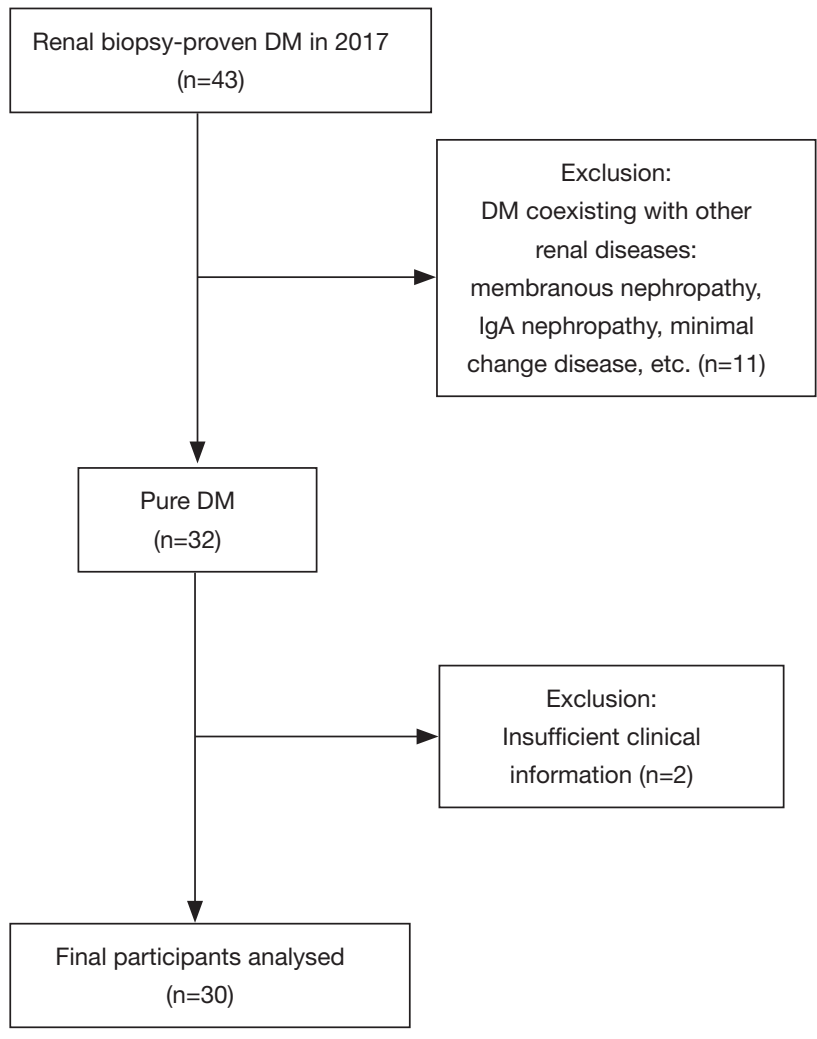

Figure 1 Flowchart for recruitment. (A) Flowchart for C-STRIDE cohort recruitment. (B) Flowchart for biopsy-proven DKD recruitment. DKD, diabetic kidney disease; DM, diabetes mellitu.

\section{Clinical evaluation}

Based on the previous research, estimated glomerular filtration rate (eGFR) was calculated (21). According to American Diabetes Association criteria, the albuminuria categorization status was categorized as described previously (22). Subgroup analysis was carried out to compare the level of sCD90 in different albuminuria statuses. The eGFR slope, and a composite endpoint of ESRD or $40 \%$ reduction of eGFR, were used to evaluate the renal progression of DKD. To estimate eGFR slope, the linear regression model was applied with no less than two eGFR data.

\section{Detection of sCD90 and membrane-bound CD90}

Soluble CD90 in urine and plasma concentration was tested with Human CD90 Immunoassay Quantikine ELISA following the manufacturer's instructions (Biomatik, Canada). Urinary sCD90 level was normalized to urine creatinine concentration, indicated as urinary sCD90/Cr. $\log _{2}$ transformed urinary sCD90/Cr and plasma sCD90 
Table 1 General data of patients from C-STRIDE cohort

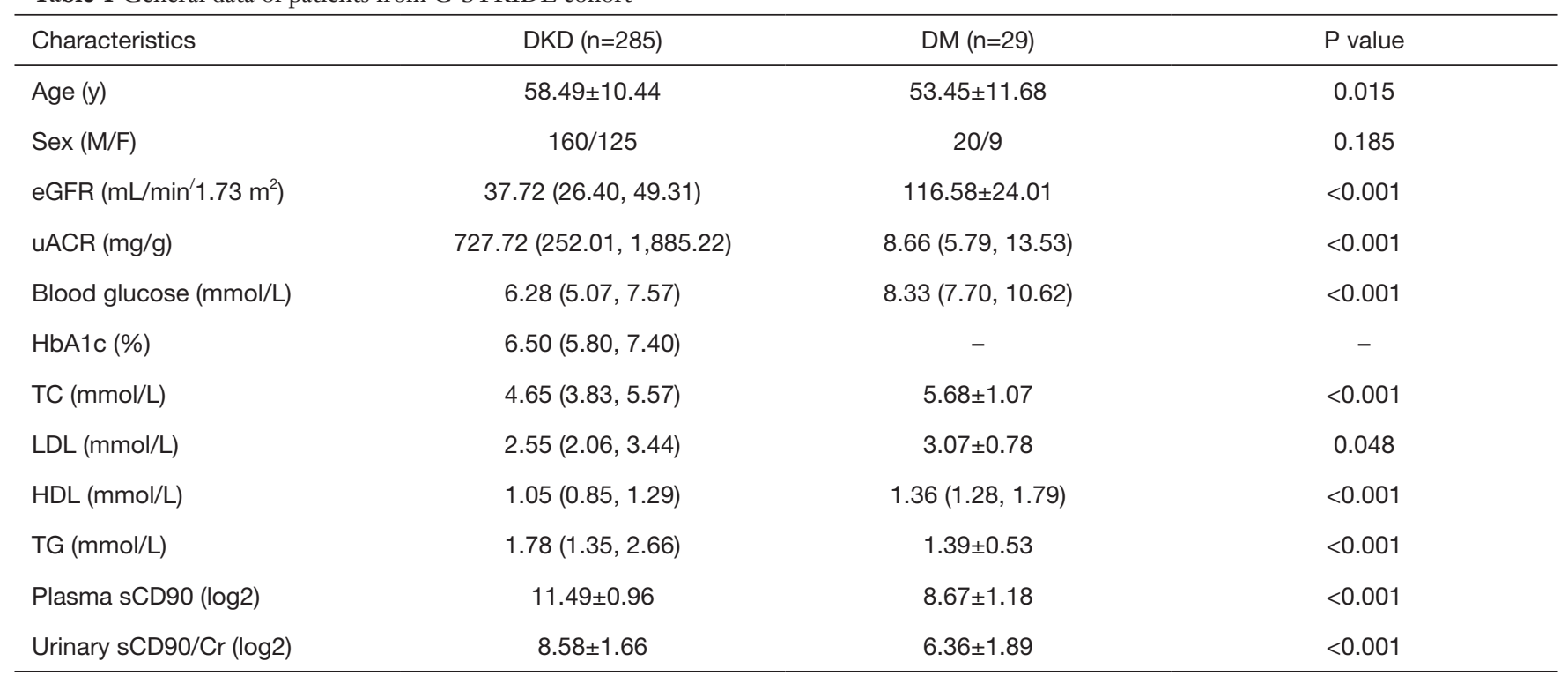

DKD, diabetic kidney disease; eGFR, estimated glomerular filtration rate; HbA1c, Hemoglobin A1c; HDL, high density lipoprotein; LDL, low density lipoprotein; NA, not available; TC, total cholesterol; TG, total triglyceride; uACR, urine albumin-to-creatinine ratio.

were used for all analyses.

To assess the expression of CD90 in kidney, we performed immunohistochemistry. Briefly, paraffinembedded renal sections were deparaffinized, rehydrated and heat mediated antigen retrieval was performed with citrate buffer ( $\mathrm{pH}$ 6.2). Sections were blocked with $3 \%$ BSA buffer and stained with a monoclonal antibody against CD90 (Abcam, Cambridge, MA; 1:200 dilution). The stained sections were then incubated with peroxidaseconjugated secondary antibodies (ZSGB-BIO, China) and developed using DAB substrate (Millipore, Bedford, MA, USA). Image-Pro Plus was used to quantify the value of mean optical density.

\section{Statistical analysis}

For continuous variables, mean $\pm \mathrm{SD}$ was used to express normally distributed, and median and interquartile range was employed to show non-normally distributed data. For categorical variables, data were expressed as absolute frequencies or percentages. Log2-transformed was used for data normalization. To compare the clinical parameters of two groups, an unpaired Student's $t$-test or Chi-square test was used. For multiple comparisons, one-way analysis of variance (ANOVA) followed by Bonferroni correction was used. Pearson's or Spearman's test was explored for the correlation analysis. Cox regression analysis was performed to identify independent predictors of composite endpoints. Those factors, significantly associated with composite endpoints in univariable Cox regression $(\mathrm{P}<0.05)$, were entered in the multivariable Cox regression model. Results were expressed as hazard ratio (HR) with $95 \%$ confidence interval (CI). Statistical analyses were performed with SPSS statistical software (version 24.0; Chicago). The cut-off of $P$ value is 0.05 .

\section{Results}

\section{General data of patients with DKD}

Two hundred and eighty-five patients with DKD were enrolled from the C-STRIDE cohort. Among them, 160 were male and 125 were female, with an age of $58.49 \pm 10.44$ years at sampling. The eGFR and uACR levels in these DKD patients were $37.72(26.40,49.31) \mathrm{mL} / \mathrm{min} / 1.73 \mathrm{~m}^{2}$ and 727.72 (252.01, $1,885.22) \mathrm{mg} / \mathrm{g}$, respectively. While in DM patients without kidney injuries, eGFR was $116.58 \pm 24.01 \mathrm{~mL} / \mathrm{min} / 1.73 \mathrm{~m}^{2}$ and uACR was $8.66(5.79,13.53) \mathrm{mg} / \mathrm{g}$. The demographic and laboratory data at baseline are shown in Table 1 .

To evaluate the expression of CD90 in renal biopsy tissues, we enrolled 30 patients with renal-biopsy confirmed DKD. Among these 30 patients, 22 were male and 8 were female, with an age of $51.23 \pm 9.75$ years at the time of renal biopsy. Most patients showed moderate to severe renal 
Table 2 General data of patients with biopsy-proven DKD

\begin{tabular}{|c|c|}
\hline Parameters & DKD \\
\hline Number of patients & 30 \\
\hline Age $($ mean $\pm S D)$ & $51.23 \pm 9.75$ \\
\hline $\operatorname{Sex}(M / F)$ & $22 / 8$ \\
\hline Diabetes history (years) (median, IQR) & $10(8,15)$ \\
\hline Type of diabetes (type 1/2) & $(0 / 30)$ \\
\hline $\operatorname{Scr}(\mu \mathrm{mol} / \mathrm{L})$ (median, IQR) & $181.98(121.78,387.17)$ \\
\hline BUN (mmol/L) (median, IQR) & $27.17(16.67,32.30)$ \\
\hline eGFR $\left(\mathrm{mL} / \mathrm{min} / 1.73 \mathrm{~m}^{2}\right)($ mean $\pm \mathrm{SD})$ & $38.07 \pm 26.81$ \\
\hline Urinary protein $(g / 24$ h) (mean \pm SD) & $5.57 \pm 3.72$ \\
\hline Fasting glucose (mmol/L) (median, IQR) & $7.27(6.34,12.27)$ \\
\hline HbA1c (\%) (median, IQR) & $7.25(5.78,8.35)$ \\
\hline Total cholesterol $(\mathrm{mmol} / \mathrm{L})($ mean $\pm \mathrm{SD})$ & $5.21 \pm 2.28$ \\
\hline Triglycerides (mmol/L) (median, IQR) & $1.99(1.33,3.37)$ \\
\hline HDL-C (mmol/L) (mean \pm SD) & $1.05 \pm 0.31$ \\
\hline LDL-C (mmol/L) (mean $\pm \mathrm{SD})$ & $3.07 \pm 1.33$ \\
\hline Glomerulosclerosis (\%) (mean \pm SD) & $32.19 \pm 24.78$ \\
\hline \multicolumn{2}{|l|}{ Tubulointerstitial lesions (n) } \\
\hline Interstitial infiltration $(-/+/++/+++)$ & $0 / 2 / 7 / 21$ \\
\hline IFTA score (grade 0/1/2/3) & $0 / 3 / 7 / 20$ \\
\hline
\end{tabular}

BUN, blood urea nitrogen; DKD, diabetic kidney disease; eGFR, estimated glomerular filtration rate; $\mathrm{HbA} 1 \mathrm{c}$, hemoglobin $\mathrm{A} 1 \mathrm{c}$; HDL-C, high-density lipoprotein cholesterol; IFTA, interstitial fibrosis and tubular atrophy; IQR, interquartile range; LDL-C, low-density lipoprotein cholesterol; Scr, serum creatinine; SD, standard deviation.

dysfunction, with an eGFR of $38.07 \pm 26.81 \mathrm{~mL} / \mathrm{min} / 1.73 \mathrm{~m}^{2}$ and proteinuria of $5.57 \pm 3.72 \mathrm{~g} / 24 \mathrm{~h}$. The clinical characteristics of these patients are listed in Table 2.

\section{The levels of sCD90 in urine and plasma}

The level of urinary sCD90/Cr in DKD patients from C-STRIDE cohort was significantly higher than that in DM patients without kidney injuries and healthy controls (8.58 \pm 1.66 vs. $6.36 \pm 1.89, \mathrm{P}<0.001 ; 8.58 \pm 1.66$ vs. $7.26 \pm 1.77$, $\mathrm{P}=0.001$, Figure 2). In addition, we divided participants into 3 groups based on albuminuria status. The level of urinary sCD90/Cr in the macroalbuminuria group was significantly higher than that in normoalbuminuria group and microalbuminuria group $(8.78 \pm 1.64$ vs. $7.92 \pm 1.47, \mathrm{P}=0.035$; $8.78 \pm 1.64$ vs. $8.08 \pm 1.77, \mathrm{P}=0.020)$. In addition, $\mathrm{sCD} 90 / \mathrm{Cr}$ levels in patients with renal-biopsy confirmed DKD were also significantly higher than those in DM patients without renal involvement $(9.12 \pm 1.56$ vs. $6.36 \pm 1.89, \mathrm{P}<0.001)$.

The DKD patients had a significantly higher level of plasma sCD90 compared with DM patients without kidney injuries and healthy controls $(11.49 \pm 0.96 \mathrm{vs}$. $8.67 \pm 1.18, \mathrm{P}<0.001 ; 11.49 \pm 0.96$ vs. $6.84 \pm 1.92, \mathrm{P}<0.001)$. No significant difference was found in the levels of plasma sCD90 among normoalbuminuria, microalbuminuria and macroalbuminuria groups of DKD patients.

\section{Associations of urinary sCD90/Cr with clinical parameters}

Compared with plasma sCD90, urinary sCD90/Cr performed better correlations with clinical parameters in patients with DKD from C-STRIDE cohort. To be more specific, there were significant correlations between urinary sCD90/Cr and eGFR $(\log 2)(\mathrm{r}=-0.373, \mathrm{P}<0.001)$, uACR $(\log 2)(\mathrm{r}=0.303, \mathrm{P}<0.001)$, and serum creatinine $(\log 2)$ (Scr; $r=0.344, \mathrm{P}<0.001)$. Besides the associations with renal function, urinary sCD90/Cr had mild correlations with blood lipid levels, including low density lipoprotein $(\log 2)$ (LDL; $\mathrm{r}=0.196, \mathrm{P}=0.001)$ and total triglyceride $(\mathrm{r}=0.209$, $\mathrm{P}<0.001)$. Moreover, the levels of urinary sCD90/Cr in patients with renal-biopsy confirmed DKD also significantly correlated with eGFR $(\mathrm{r}=-0.507, \mathrm{P}=0.004)$ and urinary protein $(\mathrm{r}=0.390, \mathrm{P}=0.033)$.

\section{Associations of urinary sCD90/Cr with renal outcomes in DKD patients}

Next, we investigated the association between urinary sCD90/Cr and the annual decline in renal function, calculated as eGFR slope from baseline per year. We found that the level of urinary sCD90/Cr had a significant correlation with the eGFR slope $(r=-0.27$, $\mathrm{P}<0.001)$. Patients were divided into two groups based on the median level of baseline urinary sCD90/Cr. Renal function of patients with higher baseline urinary sCD90/ Cr level progressed significantly faster than patients with lower baseline urinary sCD90/Cr level $(-0.74 \pm 1.40 \mathrm{vs}$. $-0.07 \pm 1.08 \mathrm{~mL} / \mathrm{min} / 1.73 \mathrm{~m}^{2}$ per month, $\left.\mathrm{P}<0.001\right)$. Among the 285 patients, 96 patients progressed to ESRD, and a total of 121 patients reached the composite endpoint during a median follow-up duration of 15 (IQR, 8 to 28.5) months. In univariable Cox regression, uACR, baseline eGFR, high 

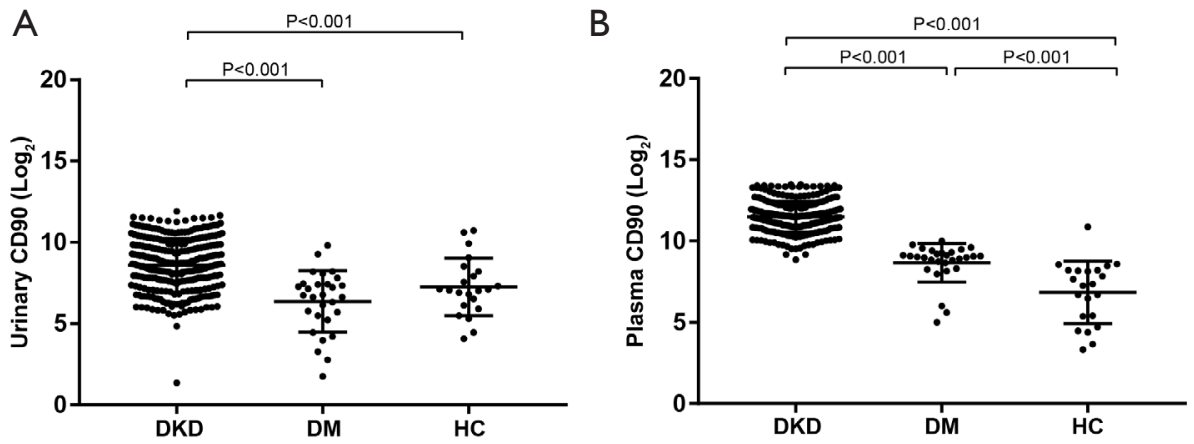

Figure 2 Soluble CD90 levels in patients with DKD. (A) The levels of urinary sCD90/Cr in DKD ( $\mathrm{n}=285$ ), DM ( $\mathrm{n}=29)$ and healthy controls $(\mathrm{n}=22)$. (B) The levels of plasma sCD90r in DKD, DM and healthy controls. Horizontal lines represent mean \pm SD. DKD, diabetic kidney disease; DM, diabetes mellitus.

Table 3 Cox regression models for the composite endpoint in DKD patients of C-STRIDE cohort

\begin{tabular}{|c|c|c|c|c|c|c|}
\hline Independent variable & \multicolumn{2}{|c|}{ Unadjusted risk } & \multicolumn{2}{|c|}{ Model 1} & \multicolumn{2}{|c|}{ Model 2} \\
\hline Age (y) & $0.98(0.97,1.00)$ & 0.012 & $1.00(0.98,1.02)$ & 0.857 & $1.00(0.98,1.02)$ & 0.866 \\
\hline Sex (male vs. female) & $1.29(0.90,1.86)$ & 0.165 & $1.76(1.16,2.65)$ & 0.007 & $1.95(1.29,2.95)$ & 0.002 \\
\hline Baseline eGFR (per mL/min'1.73 m²) & $0.98(0.97,0.99)$ & $<0.001$ & $0.99(0.98,1.00)$ & 0.131 & $1.00(0.98,1.01)$ & 0.308 \\
\hline Glucose (per mmol/L) & $0.94(0.88,1.02)$ & 0.127 & - & - & - & - \\
\hline TC (per mmol/L) & $1.01(1.00,1.02)$ & 0.158 & - & - & - & - \\
\hline $\mathrm{HDL}$ (per mmol/L) & $1.56(1.13,2.14)$ & 0.007 & $1.52(1.00,2.33)$ & 0.052 & $1.65(1.10,2.48)$ & 0.015 \\
\hline LDL (per mmol/L) & $1.43(1.27,1.61)$ & $<0.001$ & $1.22(1.03,1.45)$ & 0.021 & $1.19(1.01,1.41)$ & 0.044 \\
\hline Urinary sCD90/Cr (log2) & $1.38(1.23,1.55)$ & $<0.001$ & - & - & $1.20(1.04,1.38)$ & 0.015 \\
\hline
\end{tabular}

Cr, urine creatinine; DKD, diabetic kidney disease; eGFR, estimated glomerular filtration rate; HDL, high density lipoprotein; LDL, low density lipoprotein; TC, total cholesterol; TG, total triglyceride; UACR, urine albumin-to-creatinine ratio.

density lipoprotein (HDL), LDL, and plasma sCD90 and urinary sCD90/Cr were significantly associated with the composite endpoint. Multivariable Cox regression analysis (Table 3) showed that urinary sCD $90 / \mathrm{Cr}$, but not plasma sCD90, was independently associated with the composite endpoint, after adjusting for age, sex, baseline eGFR, uACR, HDL and LDL.

\section{Immunobistochemical staining of CD90 in renal biopsy tissue}

Renal biopsies from patients with DKD showed significantly less intense staining of CD90 in the tubulointerstitium than healthy controls (Figure $3 A, B, C$ ). However, there was no significant difference in CD90 levels in the glomeruli between DKD patients and healthy controls. The tubulointerstitial CD90 expression significantly correlated with $\mathrm{Scr}(\mathrm{r}=-0.559, \mathrm{P}=0.001)$ and eGFR ( $\mathrm{r}=0.538, \mathrm{P}=0.002)$ at the time of biopsy. Besides, tubulointerstitial CD90 expression showed significant correlations with the severity of IFTA $(\mathrm{r}=-0.3, \mathrm{P}=0.047)$ and the percentage of glomerulosclerosis $(\mathrm{r}=-0.423$, $\mathrm{P}=0.02)$. However, there was no significant correlation between tubulointerstitial CD90 expression and interstitial 
A

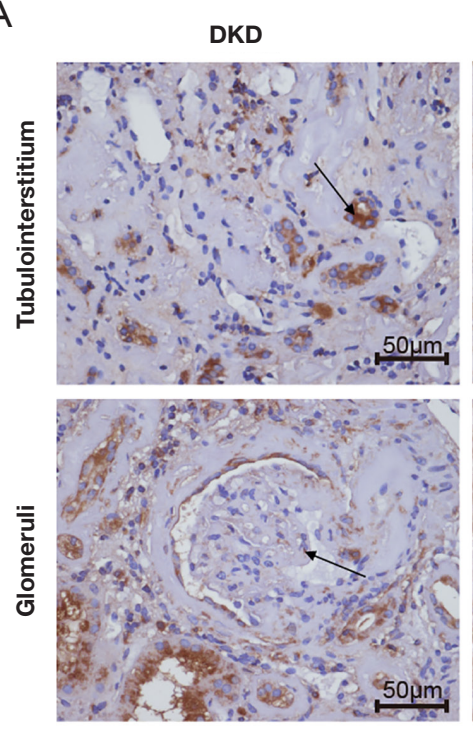

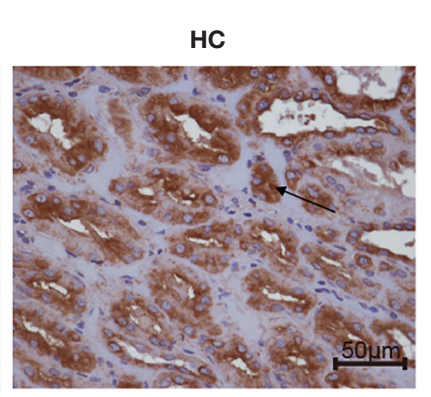

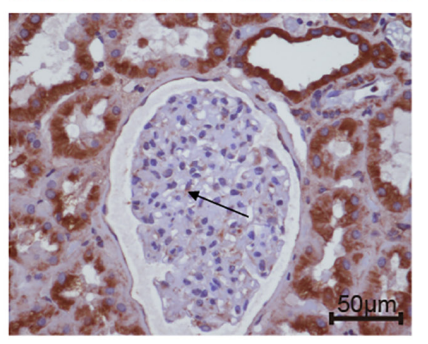

B

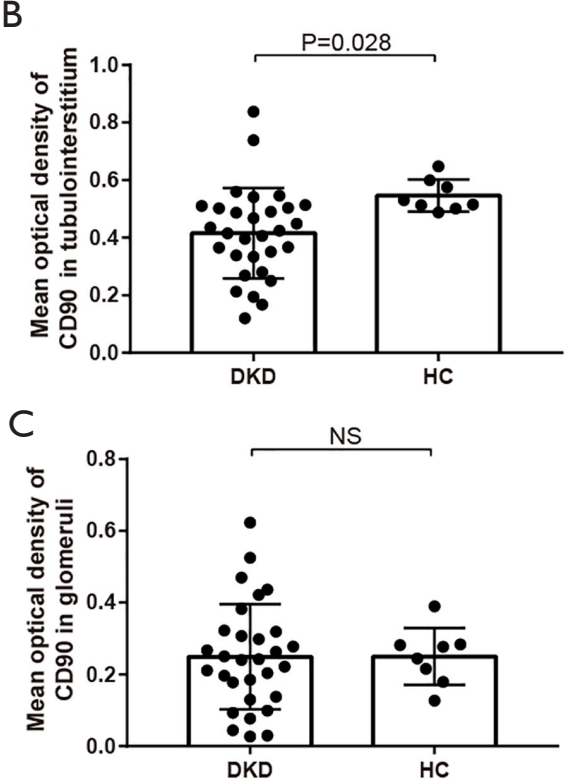

Figure 3 CD90 in renal biopsies from patients with biopsy-proven DKD. (A) Representative images of CD90 staining in human renal cortical tissue, in the tubulointerstitium and glomeruli from DKD $(n=20)$ and controls $(n=8)$. 400x magnification. Arrow: CD90. (B) Tubulointerstitial CD90 staining in DKD patients was significantly higher than that in controls. (C) Glomerular CD90 staining in DKD patients and controls. Horizontal lines represent mean \pm SD. DKD, diabetic kidney disease.

inflammation ( $\mathrm{r}=-0.171, \mathrm{P}=0.252)$. Next, we investigated the association between CD90 expression in renal biopsy and the urinary sCD90/Cr levels. Correlation analyses revealed that urinary sCD90/Cr negatively correlated with tubulointerstitial CD90 expression ( $\mathrm{r}=-0.399, \mathrm{P}=0.029$ ), while urinary sCD90/Cr did not correlate with glomerular CD90 expression in kidneys $(\mathrm{r}=-0.320, \mathrm{P}=0.084)$. Intrarenal CD90 mRNA expression was extracted from transcriptomic data (Figure 4A,B; Nephroseq, University of Michigan, Ann Arbor, MI). Consistent with the immunohistochemical results, there was a 3.213 -fold decrease $\left(\mathrm{P}=1.04 \times 10^{-4}\right)$ in tubulointerstitial CD90 mRNA expression in patients with DKD ( $\mathrm{n}=10)$ as compared with healthy living donors $(\mathrm{n}=12)$. Tubulointerstitial CD90 mRNA level significantly correlated with eGFR $(\log 2)$ at the time of biopsy $(\mathrm{r}=0.805, \mathrm{P}<0.001)$. In addition, as shown in the single cell RNA-seq (23) (Figure 4C,D,E; http://nephrocell.miktmc. org, August 2020, University of Michigan, Ann Arbor, MI, USA), CD90 was highly expressed in proximal tubular cells and fibroblasts.

\section{Discussion}

CD90, a highly conserved but somewhat mysterious molecule, exists in membrane-bound and soluble forms. It has been reported that CD90 plays a regulatory role in the differentiation of cells, especially in MSCs and MSC-like cells $(7,13,14)$. However, the role of CD90 in DKD remains largely unknown. Here, we investigated the associations of urinary and plasma sCD90 with clinical parameters as well as renal outcomes in patients with DKD. In addition, we detected the expression of CD90 in the local kidney and assessed the association between the expression of CD90 in the kidney and clinico-pathological parameters in patients with biopsy-proven DKD.

Several studies showed a markedly elevated level of sCD90 at the local site compared with the serum of the same patient $(24,25)$. In this study, we demonstrated that the plasma and urinary levels of sCD90 were significantly higher in patients with DKD compared with DM patients without kidney involvement and healthy controls. We also found that urinary sCD90 had a significant correlation with the disease severity of DKD. Cox regression analysis showed that male sex, uACR, HDL and LDL were independent risk factors for renal progression in patients with DKD, which was consistent with previous studies (26-28). More importantly, the level of urinary sCD90/Cr, but not plasma sCD90, at baseline was associated with the eGFR slope and 
A

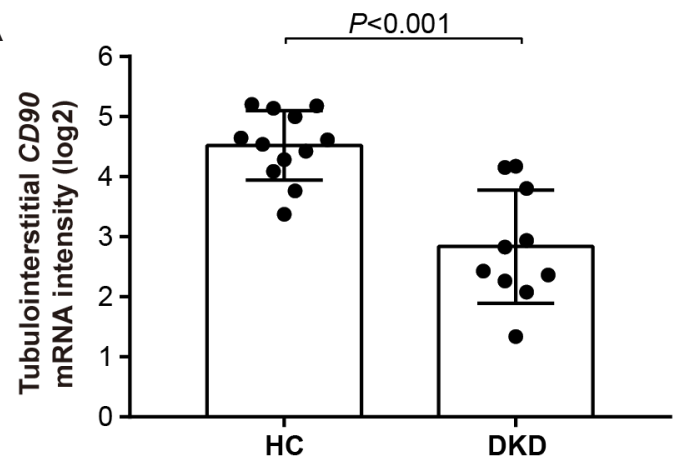

C

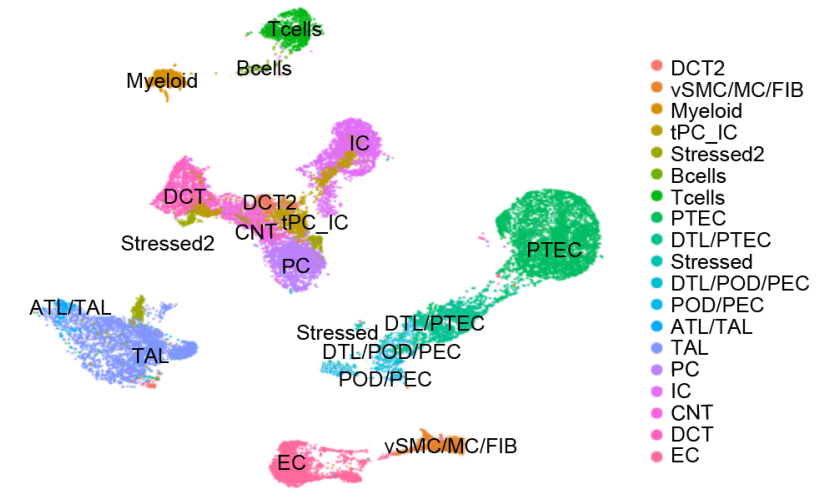

B

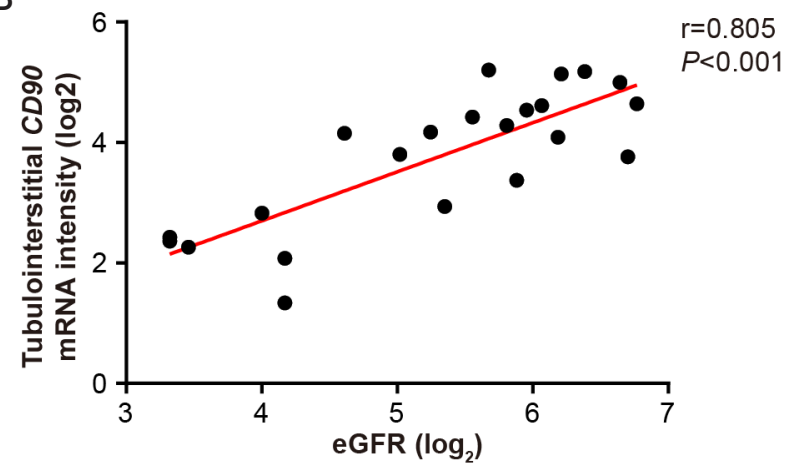

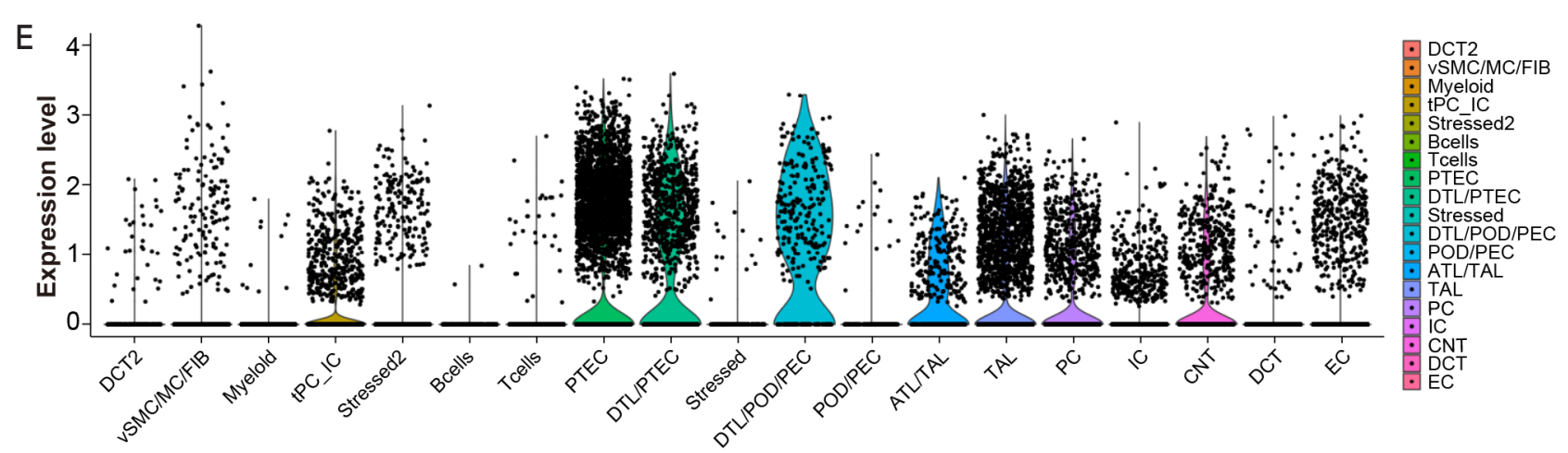

Figure 4 Bioinformatics analysis of $C D 90$ expression in renal biopsies from patients with DKD and living donors. (A) Tubulointerstitial CD90 mRNA is significantly lower in patients with DKD $(\mathrm{n}=10)$ in comparison with controls $(\mathrm{n}=12)$. Horizontal lines represent mean \pm SD. (B) Tubulointerstitial CD90 mRNA significantly correlated with eGFR $(\log 2)$. (C) UMAP plot showing the distribution of the cells in the 18 clusters resulted from the clustering of the single cell RNAseq data from living donors biopsies (n=18). (D) UMAP visualization of $C D 90$ expression. (E) Violin plot of $C D 90$ expression shows that this gene is highly expressed in PTEC_DTL, FIB, DTL/Glomerular, ATL/TAL and PTEC. DKD, diabetic kidney disease; ATL/TAL, ascending thin limb cell and thick ascending limb cell; CNT, connecting tubule; DCT, distal convoluted tubule cell; DTL, descending thin limb cell; FIB, fibroblast; IC, intercalated cell; PC, principal cell; PTEC, proximal tubule epithelial cell; tPC/IC, transitioning principal cell and intercalated cell; sSMC/MC, vascular smooth muscle cell and mesangial cell. 
was an independent predictor for renal outcomes of DKD.

Presumably, sCD90 is shed from the cell surface. In order to find the source of sCD90 in urine, we detect the expression of CD90 in the kidney. We observed a lower CD90 expression in the tubulointerstitial compartment of patients with DKD than healthy controls, while there was no significant difference in the glomerular compartment between DKD patients and healthy controls. Tubulointerstitial CD90 expression negatively correlated with urinary sCD90/Cr. Moreover, single cell RNA-seq showed that $C D 90$ mRNA was highly expressed in proximal tubular cells and fibroblasts in living donors. These results suggested that the decreased expression of tubulointerstitial CD90 may be one of the major sources for the increased urinary sCD90 in DKD. Further correlation analyses demonstrated that the expression of tubulointerstitial CD90 significantly correlated with renal function, IFTA scores and glomerulosclerosis, but not with interstitial infiltration. Several studies demonstrated the role of CD90 in lung fibrosis. Loss of CD90 expression in fibroblasts correlated with lung fibrogenesis (29). An in vitro study demonstrated that profibrotic cytokines could stimulate CD90 fibroblasts, resulting in shedding of CD90 from the cell surface and thereby switching to a more activated myofibroblast phenotype (30). Thus, we speculate that urinary sCD90 might be cleaved from the surface of intrinsic renal cells under the circumstance of DKD. On the other hand, the decrease of CD90 expression in renal cells may further lead to cell phenotype change, which may participate in extracellular matrix remodeling and tubulointerstitial fibrosis (31).

This study has several limitations. First, the sample size was relatively small, which warrants further validation in a larger cohort. Second, patients enrolled in C-STRIDE cohort did not have the renal biopsy. Thus, we are not able to evaluate the association between renal pathological changes and the outcome in these patients with DKD. Thirdly, most patients enrolled in our study had advanced kidney disease. Whether urinary sCD90 could be a useful marker at all stages of DKD still need further investigation.

In conclusion, our study demonstrated that urinary sCD90 could reflect the disease severity and serve as a predictor of renal outcome in patients with DKD.

\section{Acknowledgments}

We would like to thank Dr. Qiaoqiao Zhu (The State
University of New Jersey) for her help in polishing the language of our paper.

Funding: This study was supported by grants from the National Key Research and Development Program (No. 2016YFC1305405), two grants from the National Natural Science Fund (No. 82070748, 82090021, and 81425008), a grant from CAMS Innovation Fund for Medical Sciences (2019-I2M-5-046), and a grant by the University of Michigan Health System and Peking University Health Sciences Center Joint Institute for Translational and Clinical Research (No. BMU2017JI001). Additional support was provided by Innovation Fund for Outstanding Doctoral Candidates of Peking University Health Science Center (71013Y2029) and the George M. O'Brien Michigan Kidney Translational Core Center, funded by NIH/ NIDDK grant 2P30-DK-081943.

\section{Footnote}

Reporting Checklist: The authors have completed the STROBE reporting checklist. Available at http://dx.doi. org/10.21037/atm-20-6528

Data Sharing Statement: Available at http://dx.doi. org/10.21037/atm-20-6528

Conflicts of Interest: All authors have completed the ICMJE uniform disclosure form (available at http://dx.doi. org/10.21037/atm-20-6528). The authors have no conflicts of interest to declare.

Etbical Statement: The authors are accountable for all aspects of the work in ensuring that questions related to the accuracy or integrity of any part of the work are appropriately investigated and resolved. The study was conducted in accordance with the Declaration of Helsinki (as revised in 2013). The study was approved by the Ethics Committee of Peking University First Hospital (Beijing, China) (No.: 2011[363]; 2017[1280]) and informed consent was taken from all the patients.

Open Access Statement: This is an Open Access article distributed in accordance with the Creative Commons Attribution-NonCommercial-NoDerivs 4.0 International License (CC BY-NC-ND 4.0), which permits the noncommercial replication and distribution of the article with the strict proviso that no changes or edits are made and the 
original work is properly cited (including links to both the formal publication through the relevant DOI and the license). See: https://creativecommons.org/licenses/by-nc-nd/4.0/.

\section{References}

1. National Kidney Foundation. KDOQI clinical practice guideline for diabetes and CKD: 2012 Update. Am J Kidney Dis 2012;60:850-86.

2. de Boer IH, Rue TC, Hall YN, et al. Temporal trends in the prevalence of diabetic kidney disease in the United States. JAMA 2011;305:2532-9.

3. Liu ZH. Nephrology in china. Nat Rev Nephrol 2013;9:523-8.

4. Remuzzi G, Macia M, Ruggenenti P. Prevention and treatment of diabetic renal disease in type 2 diabetes: the BENEDICT study. J Am Soc Nephrol 2006;17:S90-7.

5. Reidy K, Kang HM, Hostetter T, et al. Molecular mechanisms of diabetic kidney disease. J Clin Invest 2014;124:2333-40.

6. Craig W, Kay R, Cutler RL, et al. Expression of Thy1 on human hematopoietic progenitor cells. J Exp Med 1993;177:1331-42.

7. Crawford JM, Barton RW. Thy-1 glycoprotein: structure, distribution, and ontogeny. Lab Invest 1986;54:122-35.

8. Schmidt M, Gutknecht D, Simon JC, et al. Controlling the balance of fibroblast proliferation and differentiation: impact of Thy-1. J Invest Dermatol 2015;135:1893-902.

9. Vitetta ES, Boyse EA, Uhr JW. Isolation and characterization of a molecular complex containing Thy1 antigen from the surface of murine thymocytes and $T$ cells. Eur J Immunol 1973;3:446-53.

10. Wetzel A, Chavakis T, Preissner KT, et al. Human Thy-1 (CD90) on activated endothelial cells is a counterreceptor for the leukocyte integrin Mac-1 (CD11b/CD18). J Immunol 2004;172:3850-9.

11. Saalbach A, Wetzig T, Haustein UF, et al. Detection of human soluble Thy-1 in serum by ELISA. Fibroblasts and activated endothelial cells are a possible source of soluble Thy-1 in serum. Cell Tissue Res 1999;298:307-15.

12. Bradley JE, Ramirez G, Hagood JS. Roles and regulation of Thy-1, a context-dependent modulator of cell phenotype. Biofactors 2009;35:258-65.

13. Sanders YY, Kumbla P, Hagood JS. Enhanced myofibroblastic differentiation and survival in Thy1(-) lung fibroblasts. Am J Respir Cell Mol Biol 2007;36:226-35.
14. Picke AK, Campbell GM, Blüher M, et al. Thy-1 (CD90) promotes bone formation and protects against obesity. Sci Transl Med 2018;10:eaao6806.

15. El Agha E, Kramann R, Schneider RK, et al. Mesenchymal stem cells in fibrotic disease. Cell Stem Cell 2017;21:166-77.

16. Gao B, Zhang L, Wang H, et al. Chinese cohort study of chronic kidney disease: design and methods. Chin Med J (Engl) 2014;127:2180-5.

17. Carville S, Wonderling D, Stevens P, et al. Early identification and management of chronic kidney disease in adults: summary of updated NICE guidance. BMJ 2014;349:g4507.

18. American Diabetes Association. Standards of medical care in diabetes. Diabetes Care 2005;28:S4-36.

19. Li XQ, Chang DY, Chen M, et al. Complement activation in patients with diabetic nephropathy. Diabetes Metab 2019;45:248-53.

20. Tervaert TW, Mooyaart AL, Amann K, et al. Pathologic classification of diabetic nephropathy. J Am Soc Nephrol 2010;21:556-63.

21. Ma YC, Zuo L, Chen JH, et al. Modified glomerular filtration rate estimating equation for Chinese patients with chronic kidney disease. J Am Soc Nephrol 2006;17:2937-44.

22. Tuttle KR, Bakris GL, Bilous RW, et al. Diabetic kidney disease: a report from an ADA Consensus Conference. Diabetes Care 2014;37:2864-83.

23. Nephrocell. Ann Arbor, MI: University of Michigan; Available online: http://nephrocell.miktmc.org

24. Kollert F, Christoph S, Probst C, et al. Soluble CD90 as a potential marker of pulmonary involvement in systemic sclerosis. Arthritis Care Res (Hoboken) 2013;65:281-7.

25. Almqvist $P$, Carlsson SR. Characterization of a hydrophilic form of Thy-1 purified from human cerebrospinal fluid. J Biol Chem 1988;263:12709-15.

26. Jardine MJ, Hata J, Woodward M, et al. Prediction of kidney-related outcomes in patients with type 2 diabetes. Am J Kidney Dis 2012;60:770-8.

27. Moore KJ, Fisher EA. Dysfunctional HDL takes its toll in chronic kidney disease. Immunity 2013;38:628-30.

28. Wu L, Li XQ, Chang DY, et al. Associations of urinary epidermal growth factor and monocyte chemotactic protein-1 with kidney involvement in patients with diabetic kidney disease. Nephrol Dial Transplant 2020;35:291-7.

29. Tan C, Jiang M, Wong SS, et al. Soluble Thy-1 reverses lung fibrosis via its integrin-binding motif. JCI Insight 
2019;4:e131152.

30. Ramírez G, Hagood JS, Sanders Y, et al. Absence of Thy-1 results in TGF- $\beta$ induced MMP-9 expression and confers a profibrotic phenotype to human lung fibroblasts. Lab
Invest 2011;91:1206-18.

31. Duffield JS. Cellular and molecular mechanisms in kidney fibrosis. J Clin Invest 2014;124:2299-306.

Cite this article as: Wu L, Chang DY, Zhang LX, Chen M, Zhao MH. Urinary soluble CD90 predicts renal prognosis in patients with diabetic kidney disease. Ann Transl Med 2021;9(4):282. doi: 10.21037/atm-20-6528 